\title{
STRONG CONVERGENCE OF MODIFIED NOOR ITERATIONS
}

\author{
YONGFU SU AND XIAOLONG QIN
}

Received 16 December 2005; Revised 24 February 2006; Accepted 12 March 2006

In this paper, strong convergence theorem is obtained for the modified Noor iterations in the framework of uniformly smooth Banach spaces. Our results extend and improve the recent ones announced by Wittman, $\mathrm{Kim}, \mathrm{Xu}$, and some others.

Copyright (c) 2006 Hindawi Publishing Corporation. All rights reserved.

\section{Introduction and preliminaries}

Let $E$ be a real Banach space, $C$ a nonempty closed convex subset of $E$, and $T: C \rightarrow C$ a mapping. Recall that $T$ is nonexpansive if

$$
\|T x-T y\| \leq\|x-y\|, \quad \forall x, y \in C
$$

A point $x \in C$ is a fixed point of $T$ provided $T x=x$. Denote by $F(T)$ the set of fixed points of $T$; that is, $F(T)=\{x \in C: T x=x\}$. It is assumed throughout the paper that $T$ is a nonexpansive mapping such that $F(T) \neq \varnothing$.

One classical way to study nonexpansive mappings is to use contractions to approximate a nonexpansive mapping $[1,9]$. More precisely, take $t \in(0,1)$ and define a contraction $T_{t}: C \rightarrow C$ by

$$
T_{t} x=t u+(1-t) T x, \quad x \in C
$$

where $u \in C$ is a fixed point. Banach's contraction mapping principle guarantees that $T_{t}$ has a unique fixed point $x_{t}$ in $C$. It is unclear, in general, what the behavior of $x_{t}$ is as $t \rightarrow 0$, even if $T$ has a fixed point. However, in the case of $T$ having a fixed point, Browder [1] proved that if $E$ is a Hilbert space, then $x_{t}$ does converge strongly to the fixed point of $T$ that is nearest to $u$. Reich [9] extended Browder's results to the setting of Banach spaces and proved that if $E$ is a uniformly smooth Banach space, then $x_{t}$ converges strongly to a fixed point of $T$ and the limit defines the (unique) sunny nonexpansive retraction from $C$ onto $F(T)$. 
Halpern [5] firstly introduced this iteration scheme:

$$
x_{0}=x \in C \quad \text { arbitrarily, } \quad x_{n+1}=\alpha_{n} u+\left(1-\alpha_{n}\right) T x_{n} ;
$$

see also Browder [2]. He pointed out that the conditions $\lim _{n \rightarrow \infty} \alpha_{n}=0$ and $\sum_{n=1}^{\infty} \alpha_{n}=\infty$ are necessary in the sense that if the iteration scheme (1.3) converges to a fixed point of $T$, then these conditions must be satisfied. Ten years later, Lions [7] investigated the general case in Hilbert space under the conditions

$$
\lim _{n \rightarrow \infty} \alpha_{n}=0, \quad \sum_{n=1}^{\infty} \alpha_{n}=\infty, \quad \lim _{n \rightarrow \infty} \frac{\alpha_{n}-\alpha_{n+1}}{\alpha_{n+1}^{2}}=0
$$

on the parameters. However, Lions' conditions on the parameters were more restrictive and did not include the natural candidate $\left\{\alpha_{n}=1 / n\right\}$. Reich [9] gave the iteration scheme (1.3) in the case when $E$ is uniformly smooth and $\alpha_{n}=n^{-\delta}$ with $0<\delta<1$.

Wittmann [11] studied the iteration scheme (1.3) in the case when $E$ is a Hilbert space and $\left\{\alpha_{n}\right\}$ satisfies

$$
\lim _{n \rightarrow \infty} \alpha_{n}=0, \quad \sum_{n=1}^{\infty} \alpha_{n}=\infty, \quad \sum_{n=1}^{\infty}|| \alpha_{n+1}-\alpha_{n} \|<\infty .
$$

Reich [10] obtained a strong convergence of the iterates (1.3) with two necessary and decreasing conditions on parameters for convergence in the case when $E$ is uniformly smooth with a weakly continuous duality mapping.

This paper introduces the composite iteration scheme:

$$
\begin{array}{ll}
w_{n}=\delta_{n} x_{n}+\left(1-\delta_{n}\right) T x_{n}, & z_{n}=\gamma_{n} x_{n}+\left(1-\gamma_{n}\right) T w_{n}, \\
y_{n}=\beta_{n} x_{n}+\left(1-\beta_{n}\right) T z_{n}, & x_{n+1}=\alpha_{n} u+\left(1-\alpha_{n}\right) y_{n},
\end{array}
$$

where $u \in C$ is an arbitrary (but fixed) element in $C$, and $\left\{\alpha_{n}\right\},\left\{\beta_{n}\right\},\left\{\gamma_{n}\right\}$, and $\left\{\delta_{n}\right\}$ are sequences in $(0,1)$. We prove, under certain appropriate assumptions on the sequences $\left\{\alpha_{n}\right\},\left\{\beta_{n}\right\},\left\{\gamma_{n}\right\}$, and $\left\{\delta_{n}\right\}$ that $\left\{x_{n}\right\}$ defined by (1.6) converges to a fixed point of $T$.

If $\delta_{n}=1$ in (1.6), then iterative scheme (1.6) is a modified Ishikawa iteration:

$$
z_{n}=\gamma_{n} x_{n}+\left(1-\gamma_{n}\right) T x_{n}, \quad y_{n}=\beta_{n} x_{n}+\left(1-\beta_{n}\right) T z_{n}, \quad x_{n+1}=\alpha_{n} u+\left(1-\alpha_{n}\right) y_{n} .
$$

If $\gamma_{n}=1 \mathrm{in}(1.6)$, then iterative scheme (1.6) is a modified Mann iteration:

$$
y_{n}=\beta_{n} x_{n}+\left(1-\beta_{n}\right) T x_{n}, \quad x_{n+1}=\alpha_{n} u+\left(1-\alpha_{n}\right) y_{n} .
$$

If $\gamma_{n}=1$ and $\beta_{n}=0$ in (1.6), then iterative scheme (1.6) is a usual Halpern iteration defined by (1.3).

$\mathrm{Kim}$ and $\mathrm{Xu}[6]$ proved the iteration scheme (1.8) converges to fixed point of $T$ in uniformly smooth Banach spaces.

It is our purpose in this paper to introduce composite iteration scheme (1.6) for approximating a fixed point of nonexpansive mappings in the framework of uniformly 
smooth Banach spaces; we establish the strong convergence of the composite iteration scheme $\left\{x_{n}\right\}$ defined by (1.6). The results improve and extend results of Kim and Xu [6], Wittmann [11], and others.

Let $E$ be a real Banach space and let $J$ denote the normalized duality mapping from $E$ into $2^{E^{*}}$ given by

$$
J(x)=\left\{f \in E^{*}:\langle x, f\rangle=\|x\|^{2}=\|f\|^{2}\right\}, \quad x \in E,
$$

where $E^{*}$ denotes the dual space of $E$ and $\langle\cdot, \cdot\rangle$ denotes the generalized duality pairing.

The norm of $E$ is said to be Gâteaux differentiable (and $E$ is said to be smooth) if

$$
\lim _{t \rightarrow 0} \frac{\|x+t y\|-\|x\|}{t}
$$

exists for each $x, y$ in its unit sphere $U=\{x \in E:\|x\|=1\}$. It is said to be uniformly Fréchet differentiable (and $E$ is said to be uniformly smooth) if the limit in (1.10) is attained uniformly for $(x, y) \in U \times U$.

We need the following definitions and lemmas for the proof of our main results.

Lemma 1.1. A Banach space $E$ is uniformly smooth if and only if the duality map $J$ is singlevalued and norm-to-norm uniformly continuous on bounded sets of E.

LemMa 1.2. In a Banach space E, there holds the inequality

$$
\|x+y\|^{2} \leq\|x\|^{2}+2\langle y, j(x+y)\rangle, \quad x, y \in E,
$$

where $j(x+y) \in J(x+y)$.

Recall that if $C$ and $D$ are nonempty subsets of a Banach space $E$ such that $C$ is nonempty closed convex and $D \subset C$, then a map $Q: C \rightarrow D$ is sunny [3,8] provided $Q(x+t(x-Q(x)))=Q(x)$ for all $x \in C$ and $t \geq 0$ whenever $x+t(x-Q(x)) \in C$. A sunny nonexpansive retraction is a sunny retraction, which is also nonexpansive. Sunny nonexpansive retractions play an important role in our argument. They are characterized as follows $[3,4,8]$ : if $E$ is a smooth Banach space, then $Q: C \rightarrow D$ is a sunny nonexpansive retraction if and only if there holds the inequality

$$
\langle x-Q x, J(y-Q x)\rangle \leq 0, \quad \forall x \in C, y \in D .
$$

Reich [9] showed that if $E$ is uniformly smooth and if $D$ is the fixed point set of a nonexpansive mapping from $C$ into itself, then there is a sunny nonexpansive retraction from $C$ onto $D$ and it can be constructed as follows.

Lemma 1.3 (see Reich [9]). Let E be a uniformly smooth Banach space and let $T: C \rightarrow C$ be a nonexpansive mapping with a fixed point $x_{t} \in C$ of the contraction $C \ni x \mapsto t u+(1-t) t x$ that converges strongly as $t \rightarrow 0$ to a fixed point of $T$. Define $Q: C \rightarrow F(T)$ by $Q u=s-$ $\lim _{t \rightarrow 0} x_{t}$. Then $Q$ is the unique sunny nonexpansive retract from $C$ onto $F(T)$; that is, $Q$ satisfies the property

$$
\langle u-Q u, J(z-Q u)\rangle \leq 0, \quad u \in C, z \in F(T) .
$$


Lemma 1.4 (see $\mathrm{Xu}[12,13])$. Let $\left\{\alpha_{n}\right\}_{n=0}^{\infty}$ be a sequence of nonnegative real numbers satisfying the property

$$
\alpha_{n+1} \leq\left(1-\gamma_{n}\right) \alpha_{n}+\gamma_{n} \sigma_{n}, \quad n \geq 0,
$$

where $\left\{\gamma_{n}\right\}_{n=0}^{\infty} \subset(0,1)$ and $\left\{\sigma_{n}\right\}_{n=0}^{\infty}$ such that

(i) $\lim _{n \rightarrow \infty} \gamma_{n}=0$ and $\sum_{n=0}^{\infty} \gamma_{n}=\infty$,

(ii) either $\limsup _{n \rightarrow \infty} \sigma_{n} \leq 0$ or $\sum_{n=0}^{\infty}\left|\gamma_{n} \sigma_{n}\right|<\infty$.

Then $\left\{\alpha_{n}\right\}_{n=0}^{\infty}$ converges to zero.

\section{Main results}

Theorem 2.1. Let $C$ be a closed convex subset of a uniformly smooth Banach space $E$ and let $T: C \rightarrow C$ be a nonexpansive mapping such that $F(T) \neq \varnothing$. Given a point $u \in C$, the initial guess $x_{0} \in C$ is chosen arbitrarily, and given sequences $\left\{\alpha_{n}\right\}_{n=0}^{\infty},\left\{\beta_{n}\right\}_{n=0}^{\infty}$ in $(0,1)$ and $\left\{\gamma_{n}\right\}_{n=0}^{\infty},\left\{\delta_{n}\right\}_{n=0}^{\infty}$ in $[0,1]$, the following conditions are satisfied:

(i) $\sum_{n=0}^{\infty} \alpha_{n}=\infty, \alpha_{n} \rightarrow 0$;

(ii) $\beta_{n}+\left(1+\beta_{n}\right)\left(1-\gamma_{n}\right)\left(2-\delta_{n}\right) \in[0, a)$ for some $a \in(0,1)$;

(iii) $\sum_{n=0}^{\infty}\left|\alpha_{n+1}-\alpha_{n}\right|<\infty, \quad \sum_{n=0}^{\infty}\left|\beta_{n+1}-\beta_{n}\right|<\infty, \quad \sum_{n=0}^{\infty}\left|\gamma_{n+1}-\gamma_{n}\right|<\infty$, and $\sum_{n=0}^{\infty}$ $\left|\delta_{n+1}-\delta_{n}\right|<\infty$.

Let $\left\{x_{n}\right\}_{n=1}^{\infty}$ be a composite process defined by

$$
\begin{array}{ll}
w_{n}=\delta_{n} x_{n}+\left(1-\delta_{n}\right) T x_{n}, & z_{n}=\gamma_{n} x_{n}+\left(1-\gamma_{n}\right) T w_{n}, \\
y_{n}=\beta_{n} x_{n}+\left(1-\beta_{n}\right) T z_{n}, & x_{n+1}=\alpha_{n} u+\left(1-\alpha_{n}\right) y_{n} .
\end{array}
$$

Then $\left\{x_{n}\right\}_{n=1}^{\infty}$ converges strongly to a fixed point of $T$.

Proof. First we observe that $\left\{x_{n}\right\}_{n=0}^{\infty}$ is bounded. Indeed, if we take a fixed point $p$ of $T$, note that

$$
\left\|w_{n}-p\right\| \leq \delta_{n}\left\|x_{n}-p\right\|+\left(1-\delta_{n}\right)\left\|T x_{n}-p\right\| \leq\left\|x_{n}-p\right\|
$$

It follows from (1.6) and (2.2) that

$$
\begin{aligned}
\left\|z_{n}-p\right\| & \leq \gamma_{n}\left\|x_{n}-p\right\|+\left(1-\gamma_{n}\right)\left\|T w_{n}-p\right\| \\
& \leq \gamma_{n}\left\|x_{n}-p\right\|+\left(1-\gamma_{n}\right)\left\|w_{n}-p\right\| \\
& \leq \gamma_{n}\left\|x_{n}-p\right\|+\left(1-\gamma_{n}\right)\left\|x_{n}-p\right\| \\
& \leq\left\|x_{n}-p\right\|, \\
\left\|y_{n}-p\right\| & \leq \beta_{n}\left\|x_{n}-p\right\|+\left(1-\beta_{n}\right)\left\|T z_{n}-p\right\| \\
& \leq \beta_{n}\left\|x_{n}-p\right\|+\left(1-\beta_{n}\right)\left\|z_{n}-p\right\| \\
& \leq \beta_{n}\left\|x_{n}-p\right\|+\left(1-\beta_{n}\right)\left\|x_{n}-p\right\| \\
& \leq\left\|x_{n}-p\right\| .
\end{aligned}
$$


Therefore

$$
\begin{aligned}
\left\|x_{n+1}-p\right\| & \leq \alpha_{n}\|u-p\|+\left(1-\alpha_{n}\right)\left\|y_{n}-p\right\| \\
& \leq \alpha_{n}\|u-p\|+\left(1-\alpha_{n}\right)\left\|x_{n}-p\right\| \\
& \leq \max \left\{\|u-p\|,\left\|x_{n}-p\right\|\right\} .
\end{aligned}
$$

Now, an induction yields

$$
\left\|x_{n}-p\right\| \leq \max \left\{\|u-p\|,\left\|x_{0}-p\right\|\right\}, \quad n \geq 0
$$

Hence, $\left\{x_{n}\right\}$ is bounded, so are $\left\{y_{n}\right\},\left\{z_{n}\right\}$, and $\left\{w_{n}\right\}$. Next, we claim that

$$
\left\|x_{n+1}-x_{n}\right\| \longrightarrow 0
$$

In order to prove (2.6), it follows from (1.6) that

$$
x_{n+1}=\alpha_{n} u+\left(1-\alpha_{n}\right) y_{n}, \quad x_{n}=\alpha_{n-1} u+\left(1-\alpha_{n-1}\right) y_{n-1} .
$$

We have

$$
\begin{aligned}
x_{n+1}-x_{n}= & \left(1-\alpha_{n}\right)\left(1-\beta_{n}\right)\left(T z_{n}-T z_{n-1}\right)+\left(1-\alpha_{n}\right) \beta_{n}\left(x_{n}-x_{n-1}\right) \\
& +\left[\left(\beta_{n}-\beta_{n-1}\right)\left(1-\alpha_{n}\right)-\left(\alpha_{n}-\alpha_{n-1}\right) \beta_{n-1}\right]\left(x_{n-1}-T z_{n-1}\right) \\
& +\left(\alpha_{n}-\alpha_{n-1}\right)\left(u-T z_{n-1}\right) .
\end{aligned}
$$

Therefore

$$
\begin{aligned}
\left\|x_{n+1}-x_{n}\right\| \leq & \left(1-\beta_{n}\right)\left(1-\alpha_{n}\right)\left\|T z_{n}-T z_{n-1}\right\|+\left(1-\alpha_{n}\right) \beta_{n}\left\|x_{n}-x_{n-1}\right\| \\
& +\left|\left(\beta_{n}-\beta_{n-1}\right)\left(1-\alpha_{n}\right)-\left(\alpha_{n}-\alpha_{n-1}\right) \beta_{n-1}\right||| x_{n-1}-T z_{n-1} \| \\
& +\left|\alpha_{n}-\alpha_{n-1}\right||| u-T z_{n-1}|| .
\end{aligned}
$$

That is,

$$
\begin{aligned}
\left\|x_{n+1}-x_{n}\right\| \leq & \left(1-\beta_{n}\right)\left(1-\alpha_{n}\right)\left\|z_{n}-z_{n-1}\right\|+\left(1-\alpha_{n}\right) \beta_{n}\left\|x_{n}-x_{n-1}\right\| \\
& +\left|\left(\beta_{n}-\beta_{n-1}\right)\left(1-\alpha_{n}\right)-\left(\alpha_{n}-\alpha_{n-1}\right) \beta_{n-1}\right||| x_{n-1}-T z_{n-1} \| \\
& +\left|\alpha_{n}-\alpha_{n-1}\right|\left\|u-T z_{n-1}\right\| .
\end{aligned}
$$

Again, from (1.6) we obtain

$$
w_{n}=\delta_{n} x_{n}+\left(1-\delta_{n}\right) T x_{n}, \quad w_{n-1}=\delta_{n-1} x_{n-1}+\left(1-\delta_{n-1}\right) T x_{n-1} ;
$$

therefore

$$
w_{n}-w_{n-1}=\left(1-\delta_{n}\right)\left(T x_{n}-T x_{n-1}\right)+\delta_{n}\left(x_{n}-x_{n-1}\right)+\left(\delta_{n}-\delta_{n-1}\right)\left(x_{n-1}-T x_{n-1}\right),
$$


that is,

$$
\left\|w_{n}-w_{n-1}\right\| \leq\left\|x_{n}-x_{n-1}\right\|+\left|\delta_{n}-\delta_{n-1}\right||| x_{n-1}-T x_{n-1} \| .
$$

Similarly we obtain

$$
z_{n}=\gamma_{n} x_{n}+\left(1-\gamma_{n}\right) T w_{n}, \quad z_{n-1}=\gamma_{n-1} x_{n-1}+\left(1-\gamma_{n-1}\right) T w_{n-1} .
$$

Thus,

$$
z_{n}-z_{n-1}=\left(1-\gamma_{n}\right)\left(T w_{n}-T w_{n-1}\right)+\gamma_{n}\left(x_{n}-x_{n-1}\right)+\left(\gamma_{n}-\gamma_{n-1}\right)\left(x_{n-1}-T w_{n-1}\right) \text {, }
$$

that is,

$$
\begin{aligned}
\left\|z_{n}-z_{n-1}\right\| & \leq\left(1-\gamma_{n}\right)|| T w_{n}-T w_{n-1}||+\gamma_{n}|| x_{n}-x_{n-1}||+\left|\gamma_{n}-\gamma_{n-1}\right||| x_{n-1}-T w_{n-1} \| \\
& \leq\left(1-\gamma_{n}\right)|| w_{n}-w_{n-1}||+\gamma_{n}|| x_{n}-x_{n-1}||+\left|\gamma_{n}-\gamma_{n-1}\right||| x_{n-1}-T w_{n-1} \| .
\end{aligned}
$$

Now, substituting (2.13) into (2.16) we have

$$
\begin{aligned}
\left\|z_{n}-z_{n-1}\right\| \leq & \left(1-\gamma_{n}\right)|| w_{n}-w_{n-1}||+\gamma_{n}|| x_{n}-x_{n-1}||+\left|\gamma_{n}-\gamma_{n-1}\right||| x_{n-1}-T w_{n-1} \| \\
\leq & \left(1-\gamma_{n}\right)\left(|| x_{n}-x_{n-1}||+\left|\delta_{n}-\delta_{n-1}\right||| x_{n-1}-T x_{n-1}||\right) \\
& +\gamma_{n}|| x_{n}-x_{n-1}||+\left|\gamma_{n}-\gamma_{n-1}\right||| x_{n-1}-T w_{n-1} \| \\
\leq & \left\|x_{n}-x_{n-1}||+\left|\delta_{n}-\delta_{n-1}\right||| x_{n-1}-T x_{n-1}||+\left|\gamma_{n}-\gamma_{n-1}\right||| x_{n-1}-T w_{n-1}\right\| .
\end{aligned}
$$

It follows that

$$
|| z_{n}-z_{n-1} \| \leq|| x_{n}-x_{n-1}||+\left(\left|\delta_{n-1}-\delta_{n}\right|+\left|\gamma_{n-1}-\gamma_{n}\right|\right) M_{1},
$$

where $M_{1}$ is a constant such that

$$
M_{1} \geq \max \left\{\left\|x_{n-1}-T x_{n-1}\right\|,\left\|T w_{n-1}-x_{n-1}\right\|\right\} .
$$

Substituting (2.18) into (2.10), we get

$$
\begin{aligned}
\left\|x_{n+1}-x_{n}\right\| \leq & \left(1-\alpha_{n}\right)|| x_{n}-x_{n-1}||+\left(1-\alpha_{n}\right)\left(1-\beta_{n}\right)\left(\left|\delta_{n-1}-\delta_{n}\right|+\left|\gamma_{n-1}-\gamma_{n}\right|\right) M_{1} \\
& +\left|\left(\beta_{n}-\beta_{n-1}\right)\left(1-\alpha_{n}\right)-\left(\alpha_{n}-\alpha_{n-1}\right) \beta_{n-1}\right||| x_{n-1}-T z_{n-1}|| \\
& +\left|\alpha_{n}-\alpha_{n-1}\right||| u-T z_{n-1}||,
\end{aligned}
$$

that is,

$$
\begin{aligned}
\left\|x_{n+1}-x_{n}\right\| \leq & \left(1-\alpha_{n}\right)|| x_{n}-x_{n-1}|| \\
& +M\left(\left|\gamma_{n}-\gamma_{n-1}\right|+\left|\beta_{n}-\beta_{n-1}\right|+2\left|\alpha_{n}-\alpha_{n-1}\right|+\left|\delta_{n}-\delta_{n-1}\right|\right),
\end{aligned}
$$


where $M$ is a constant such that

$$
M \geq \max \left\{\left\|u-T z_{n-1}\right\|,\left\|x_{n-1}-T z_{n-1}\right\|, M_{1}\right\}
$$

for all $n$. By assumptions (i)-(iii), we have that

$$
\begin{gathered}
\lim _{n \rightarrow \infty} \alpha_{n}=0, \quad \sum_{n=1}^{\infty} \alpha_{n}=\infty, \\
\sum_{n=1}^{\infty}\left(\left|\gamma_{n}-\gamma_{n-1}\right|+\left|\beta_{n}-\beta_{n-1}\right|+2\left|\alpha_{n}-\alpha_{n-1}\right|+\left|\delta_{n}-\delta_{n-1}\right|\right)<\infty .
\end{gathered}
$$

Hence, Lemma 1.4 is applicable to (2.21) and we obtain

$$
\left\|x_{n+1}-x_{n}\right\| \longrightarrow 0
$$

On the other hand, from (1.6) and condition (i), we have

$$
\begin{aligned}
\left\|x_{n+1}-y_{n}\right\|= & \alpha_{n}\left\|u-y_{n}\right\| \longrightarrow 0, \quad \text { as } n \rightarrow \infty, \\
\left\|T x_{n}-x_{n}\right\| \leq & \left\|x_{n}-x_{n+1}\right\|+\left\|x_{n+1}-y_{n}\right\|+\left\|y_{n}-T z_{n}\right\|+\left\|T z_{n}-T x_{n}\right\| \\
\leq & \left\|x_{n}-x_{n+1}\right\|+\left\|x_{n+1}-y_{n}\right\|+\left\|y_{n}-T z_{n}\right\|+\left\|z_{n}-x_{n}\right\| \\
\leq & \left\|x_{n}-x_{n+1}\right\|+\left\|x_{n+1}-y_{n}\right\|+\beta_{n}\left\|x_{n}-T z_{n}\right\|+\left\|z_{n}-x_{n}\right\| \\
\leq & \left\|x_{n}-x_{n+1}\right\|+\left\|x_{n+1}-y_{n}\right\|+\beta_{n}\left\|x_{n}-T x_{n}\right\| \\
& +\beta_{n}\left\|T x_{n}-T z_{n}\right\|+\left\|z_{n}-x_{n}\right\| \\
\leq & \left\|x_{n}-x_{n+1}\right\|+\left\|x_{n+1}-y_{n}\right\|+\beta_{n}\left\|x_{n}-T x_{n}\right\| \\
& +\beta_{n}\left\|x_{n}-z_{n}\right\|+\left\|z_{n}-x_{n}\right\| \\
= & \left\|x_{n}-x_{n+1}\right\|+\left\|x_{n+1}-y_{n}\right\|+\beta_{n}\left\|x_{n}-T x_{n}\right\| \\
& +\left(1+\beta_{n}\right)\left\|x_{n}-z_{n}\right\| \\
\leq & \left\|x_{n}-x_{n+1}\right\|+\left\|x_{n+1}-y_{n}\right\|+\beta_{n}\left\|x_{n}-T x_{n}\right\| \\
& +\left(1+\beta_{n}\right)\left(1-\gamma_{n}\right)\left\|x_{n}-T w_{n}\right\| \\
\leq & \left\|x_{n}-x_{n+1}\right\|+\left\|x_{n+1}-y_{n}\right\|+\beta_{n}\left\|x_{n}-T x_{n}\right\| \\
& +\left(1+\beta_{n}\right)\left(1-\gamma_{n}\right)\left\|x_{n}-T x_{n}\right\|+\left(1+\beta_{n}\right)\left(1-\gamma_{n}\right)\left\|T x_{n}-T w_{n}\right\| \\
\leq & \left\|x_{n}-x_{n+1}\right\|+\left\|x_{n+1}-y_{n}\right\|+\beta_{n}\left\|x_{n}-T x_{n}\right\| \\
& +\left(1+\beta_{n}\right)\left(1-\gamma_{n}\right)\left\|x_{n}-T x_{n}\right\|+\left(1+\beta_{n}\right)\left(1-\gamma_{n}\right)\left\|x_{n}-w_{n}\right\| \\
\leq & \left\|x_{n}-x_{n+1}\right\|+\left\|x_{n+1}-y_{n}\right\|+\beta_{n}\left\|x_{n}-T x_{n}\right\| \\
& +\left(1+\beta_{n}\right)\left(1-y_{n}\right)\left\|x_{n}-T x_{n}\right\|+\left(1+\beta_{n}\right)\left(1-\gamma_{n}\right)\left(1-\delta_{n}\right)\left\|T x_{n}-x_{n}\right\| .
\end{aligned}
$$


8 Nonexpansive mapping

It follows that

$$
\begin{aligned}
& \left\{1-\left[\beta_{n}+\left(1+\beta_{n}\right)\left(1-\gamma_{n}\right)+\left(1+\beta_{n}\right)\left(1-\gamma_{n}\right)\left(1-\delta_{n}\right)\right]\right\}\left\|T x_{n}-x_{n}\right\| \\
& \leq\left\|x_{n}-x_{n+1}\right\|+\left\|x_{n+1}-y_{n}\right\| .
\end{aligned}
$$

That is,

$$
\begin{aligned}
& \left\{1-\left[\beta_{n}+\left(1+\beta_{n}\right)\left(1-\gamma_{n}\right)\left(2-\delta_{n}\right)\right]\right\}\left\|T x_{n}-x_{n}\right\| \\
& \leq\left\|x_{n}-x_{n+1}\right\|+\left\|x_{n+1}-y_{n}\right\| .
\end{aligned}
$$

From condition (ii), (2.24), and (2.25), we know

$$
\left\|T x_{n}-x_{n}\right\| \longrightarrow 0
$$

Next, we claim that

$$
\limsup _{n \rightarrow \infty}\left\langle u-q, J\left(x_{n}-q\right)\right\rangle \leq 0
$$

where $q=Q u=s-\lim _{t \rightarrow 0} z_{t}$ with $z_{t}$ being the fixed point of the contraction $z \mapsto t u+$ $(1-t) T z$.

From $z_{t}$, the fixed point equation

$$
z_{t}=t u+(1-t) T z_{t}
$$

is solved. Since $T_{t}$ defined by (1.2) is a contraction, we obtain that $T_{t}$ has a unique fixed point. Thus we have

$$
\left\|z_{t}-x_{n}\right\|=\left\|(1-t)\left(T z_{t}-x_{n}\right)+t\left(u-x_{n}\right)\right\|
$$

It follows from Lemma 1.2 that

$$
\begin{aligned}
\left\|z_{t}-x_{n}\right\|^{2} \leq & (1-t)^{2}\left\|T z_{t}-x_{n}\right\|^{2}+2 t\left\langle u-x_{n}, J\left(z_{t}-x_{n}\right)\right\rangle \\
\leq & \left(1-2 t+t^{2}\right)\left\|z_{t}-x_{n}\right\|^{2}+f_{n}(t) \\
& +2 t\left\langle u-z_{t}, J\left(z_{t}-x_{n}\right)\right\rangle+2 t\left\|z_{t}-x_{n}\right\|^{2},
\end{aligned}
$$

where

$$
f_{n}(t)=\left(2|| z_{t}-x_{n}\|+\| x_{n}-T x_{n} \|\right)\left\|x_{n}-T x_{n}\right\| \longrightarrow 0, \quad \text { as } n \longrightarrow 0 .
$$

It follows that

$$
\left\langle z_{t}-u, J\left(z_{t}-x_{n}\right)\right\rangle \leq \frac{t}{2}\left\|z_{t}-x_{n}\right\|^{2}+\frac{1}{2 t} f_{n}(t) .
$$

Letting $n \rightarrow \infty$ in (2.35) and noting (2.34) yield

$$
\limsup _{n \rightarrow \infty}\left\langle z_{t}-u, J\left(z_{t}-x_{n}\right)\right\rangle \leq \frac{t}{2} M
$$


where $M>0$ is a constant such that $M \geq\left\|z_{t}-x_{n}\right\|^{2}$ for all $t \in(0,1)$ and $n \geq 1$. Letting $t \rightarrow 0$ from (2.36), we have

$$
\limsup _{t \rightarrow 0} \limsup _{n \rightarrow \infty}\left\langle z_{t}-u, J\left(z_{t}-x_{n}\right)\right\rangle \leq 0
$$

So, for any $\epsilon>0$, there exists a positive number $\delta_{1}$ such that, for $t \in\left(0, \delta_{1}\right)$, we get

$$
\limsup _{n \rightarrow \infty}\left\langle z_{t}-u, J\left(z_{t}-x_{n}\right)\right\rangle \leq \frac{\epsilon}{2} .
$$

On the other hand, since $z_{t} \rightarrow q$ as $t \rightarrow 0$, from Lemma 1.1, there exists $\delta_{2}>0$ such that, for $t \in\left(0, \delta_{2}\right)$, we have

$$
\begin{aligned}
&\left|\left\langle u-q, J\left(x_{n}-q\right)\right\rangle-\left\langle z_{t}-u, J\left(z_{t}-x_{n}\right)\right\rangle\right| \\
& \leq\left|\left\langle u-q, J\left(x_{n}-q\right)\right\rangle-\left\langle u-q, J\left(x_{n}-z_{t}\right)\right\rangle\right| \\
& \quad+\left|\left\langle u-q, J\left(x_{n}-z_{t}\right)\right\rangle-\left\langle z_{t}-u, J\left(z_{t}-x_{n}\right)\right\rangle\right| \\
& \leq\left|\left\langle u-q, J\left(x_{n}-q\right)-J\left(x_{n}-z_{t}\right)\right\rangle\right|+\left|\left\langle z_{t}-q, J\left(x_{n}-z_{t}\right)\right\rangle\right| \\
& \leq\|u-q\||| J\left(x_{n}-q\right)-J\left(x_{n}-z_{t}\right)\left\|+|| z_{t}-q\right\||| x_{n}-z_{t} \|<\frac{\epsilon}{2} .
\end{aligned}
$$

Choosing $\delta=\min \left\{\delta_{1}, \delta_{2}\right\}$, for all $t \in(0, \delta)$, we have

$$
\left\langle u-q, J\left(x_{n}-q\right)\right\rangle \leq\left\langle z_{t}-u, J\left(z_{t}-x_{n}\right)\right\rangle+\frac{\epsilon}{2},
$$

that is,

$$
\limsup _{n \rightarrow \infty}\left\langle u-q, J\left(x_{n}-q\right)\right\rangle \leq \lim _{n \rightarrow \infty}\left\langle z_{t}-u, J\left(z_{t}-x_{n}\right)\right\rangle+\frac{\epsilon}{2} .
$$

It follows from (2.38) that

$$
\limsup _{n \rightarrow \infty}\left\langle u-q, J\left(x_{n}-q\right)\right\rangle \leq \epsilon .
$$

Since $\epsilon$ is chosen arbitrarily, we have

$$
\limsup _{n \rightarrow \infty}\left\langle u-q, J\left(x_{n}-q\right)\right\rangle \leq 0 .
$$

Finally, we show that $x_{n} \rightarrow q$ strongly and this concludes the proof. Indeed, using Lemma 1.2 again, we obtain

$$
\begin{aligned}
\left\|x_{n+1}-q\right\|^{2} & =\left\|\left(1-\alpha_{n}\right)\left(y_{n}-q\right)+\alpha_{n}(u-q)\right\|^{2} \\
& \leq\left(1-\alpha_{n}\right)^{2}\left\|y_{n}-q\right\|^{2}+2 \alpha_{n}\left\langle u-q, J\left(x_{n+1}-q\right)\right\rangle \\
& \leq\left(1-\alpha_{n}\right)\left\|x_{n}-q\right\|^{2}+2 \alpha_{n}\left\langle u-q, J\left(x_{n+1}-q\right)\right\rangle .
\end{aligned}
$$

Now we apply Lemma 1.4 and use (2.43) to see that $\left\|x_{n}-q\right\| \rightarrow 0$. 
As a corollary of Theorem 2.1, we have the following.

Corollary 2.2. Let $C$ be a closed convex subset of a uniformly smooth Banach space $E$ and let $T: C \rightarrow C$ be a nonexpansive mapping such that $F(T) \neq \varnothing$. Given a point $u \in C$, the initial guess $x_{0} \in C$ is chosen arbitrarily, and given sequences $\left\{\alpha_{n}\right\}_{n=0}^{\infty},\left\{\beta_{n}\right\}_{n=0}^{\infty}$ in $(0,1)$ and $\left\{\gamma_{n}\right\}_{n=0}^{\infty}$ in $[0,1]$, the following conditions are satisfied:

(i) $\sum_{n=0}^{\infty} \alpha_{n}=\infty, \alpha_{n} \rightarrow 0$;

(ii) $\beta_{n}+\left(1+\beta_{n}\right)\left(1-\gamma_{n}\right) \in[0, a)$ for some $a \in(0,1)$;

(iii) $\sum_{n=0}^{\infty}\left|\alpha_{n+1}-\alpha_{n}\right|<\infty, \sum_{n=0}^{\infty}\left|\beta_{n+1}-\beta_{n}\right|<\infty$, and $\sum_{n=0}^{\infty}\left|\gamma_{n+1}-\gamma_{n}\right|<\infty$.

Let $\left\{x_{n}\right\}_{n=1}^{\infty}$ be the composite process defined by (1.7).

Then $\left\{x_{n}\right\}_{n=1}^{\infty}$ converges strongly to a fixed point of $T$.

Proof. By taking $\delta_{n}=1$, we can obtain the desired conclusion.

Corollary 2.3. Let $C$ be a closed convex subset of a uniformly smooth Banach space $E$ and let $T: C \rightarrow C$ be a nonexpansive mapping such that $F(T) \neq \varnothing$. Given a point $u \in C$, the initial guess $x_{0} \in C$ is chosen arbitrarily, and given sequences $\left\{\alpha_{n}\right\}_{n=0}^{\infty},\left\{\beta_{n}\right\}_{n=0}^{\infty}$ in $(0,1)$, the following conditions are satisfied:

(i) $\sum_{n=0}^{\infty} \alpha_{n}=\infty, \alpha_{n} \rightarrow 0$;

(ii) $\beta_{n} \in[0, a)$ for some $a \in(0,1)$;

(iii) $\sum_{n=0}^{\infty}\left|\alpha_{n+1}-\alpha_{n}\right|<\infty, \sum_{n=0}^{\infty}\left|\beta_{n+1}-\beta_{n}\right|<\infty$.

Let $\left\{x_{n}\right\}_{n=1}^{\infty}$ be the composite process defined by (1.8).

Then $\left\{x_{n}\right\}_{n=1}^{\infty}$ converges strongly to a fixed point of T.

Proof. By taking $\gamma_{n}=1$, then $\left\{x_{n}\right\}_{n=1}^{\infty}$ converges strongly to a fixed point of $T$.

\section{References}

[1] F. E. Browder, Fixed-point theorems for noncompact mappings in Hilbert space, Proceedings of the National Academy of Sciences of the United States of America 53 (1965), 1272-1276.

[2] _ Convergence of approximants to fixed points of nonexpansive non-linear mappings in Banach spaces, Archive for Rational Mechanics and Analysis 24 (1967), no. 1, 82-90.

[3] R. E. Bruck Jr., Nonexpansive projections on subsets of Banach spaces, Pacific Journal of Mathematics 47 (1973), 341-355.

[4] K. Goebel and S. Reich, Uniform Convexity, Hyperbolic Geometry, and Nonexpansive Mappings, Monographs and Textbooks in Pure and Applied Mathematics, vol. 83, Marcel Dekker, New York, 1984.

[5] B. Halpern, Fixed points of nonexpanding maps, Bulletin of the American Mathematical Society. New Series 73 (1967), 957-961.

[6] T. H. Kim and H. K. Xu, Strong convergence of modified Mann iterations, Nonlinear Analysis 61 (2005), no. 1-2, 51-60.

[7] P.-L. Lions, Approximation de points fixes de contractions, Comptes Rendus Mathematique. Academie des Sciences. Paris Série A-B 284 (1977), no. 21, A1357-A1359.

[8] S. Reich, Asymptotic behavior of contractions in Banach spaces, Journal of Mathematical Analysis and Applications 44 (1973), no. 1, 57-70.

[9] __ Strong convergence theorems for resolvents of accretive operators in Banach spaces, Journal of Mathematical Analysis and Applications 75 (1980), no. 1, 287-292. 
[10] Approximating fixed points of nonexpansive mappings, Panamerican Mathematical Journal 4 (1994), no. 2, 23-28.

[11] R. Wittmann, Approximation of fixed points of nonexpansive mappings, Archiv der Mathematik 58 (1992), no. 5, 486-491.

[12] H. K. Xu, Iterative algorithms for nonlinear operators, Journal of the London Mathematical Society. Second Series 66 (2002), no. 1, 240-256.

[13] _ An iterative approach to quadratic optimization, Journal of Optimization Theory and Applications 116 (2003), no. 3, 659-678.

Yongfu Su: Department of Mathematics, Tianjin Polytechnic University,

Tianjin 300160, China

E-mail address: suyongfu@tjpu.edu.cn

Xiaolong Qin: Department of Mathematics, Tianjin Polytechnic University, Tianjin 300160, China

E-mail address: qxlxajh@163.com 


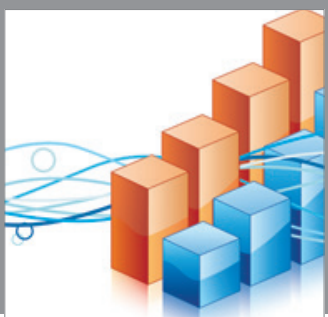

Advances in

Operations Research

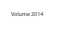

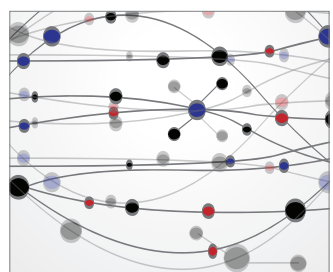

\section{The Scientific} World Journal
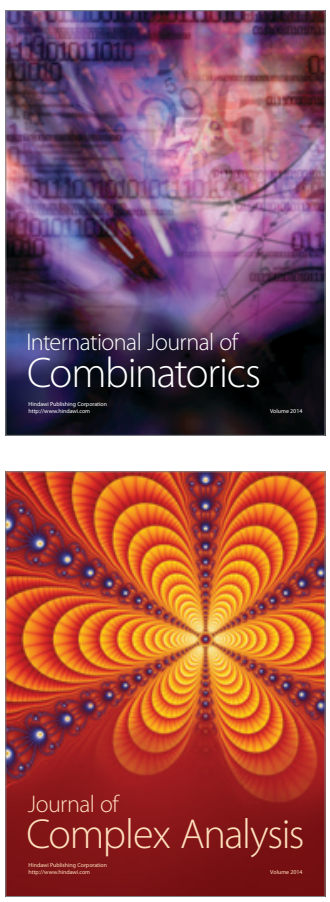

International Journal of

Mathematics and

Mathematical

Sciences
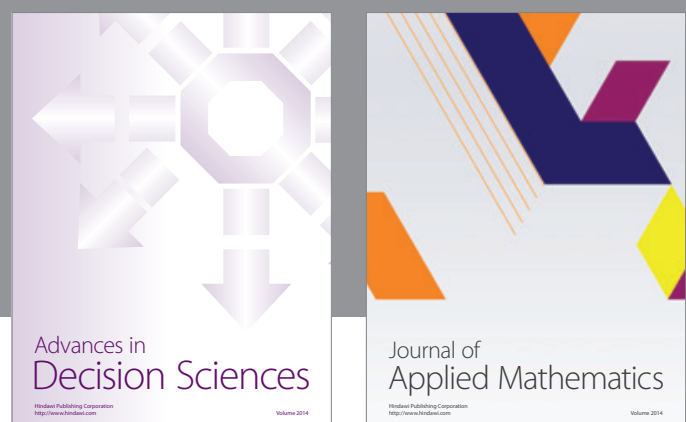

Journal of

Applied Mathematics
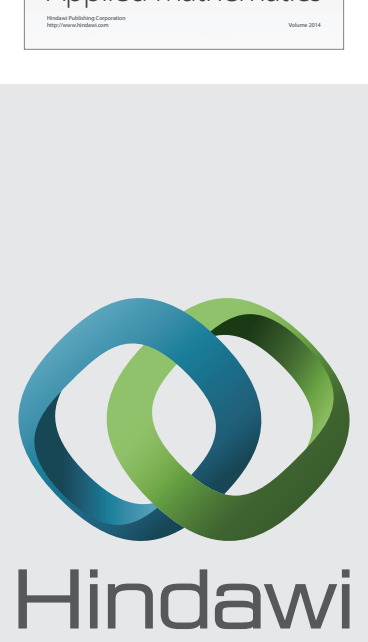

Submit your manuscripts at http://www.hindawi.com
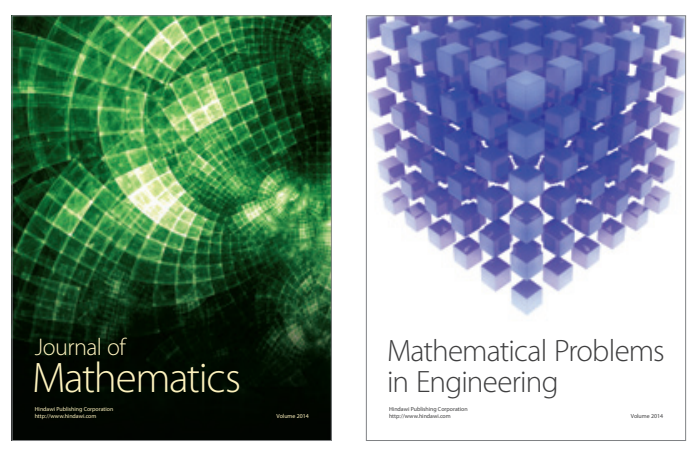

Mathematical Problems in Engineering
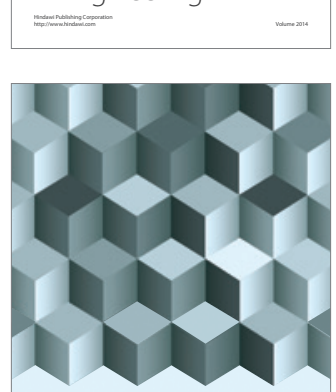

Journal of

Function Spaces
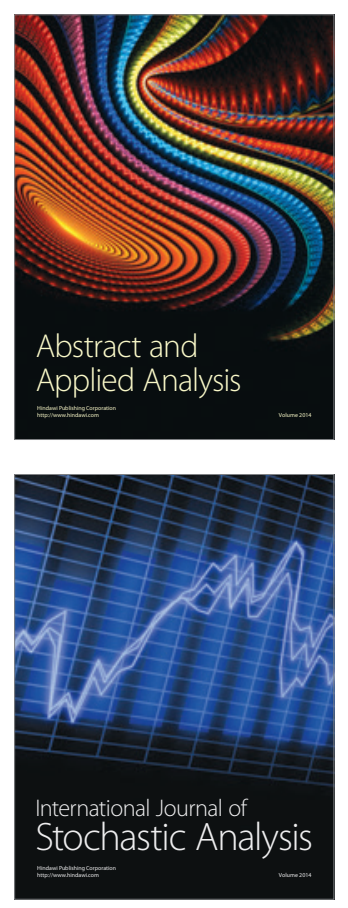

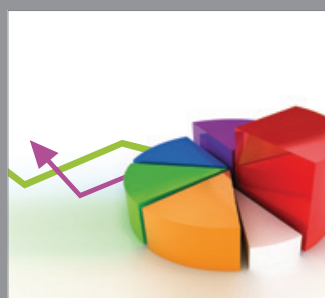

ournal of

Probability and Statistics

Promensencen
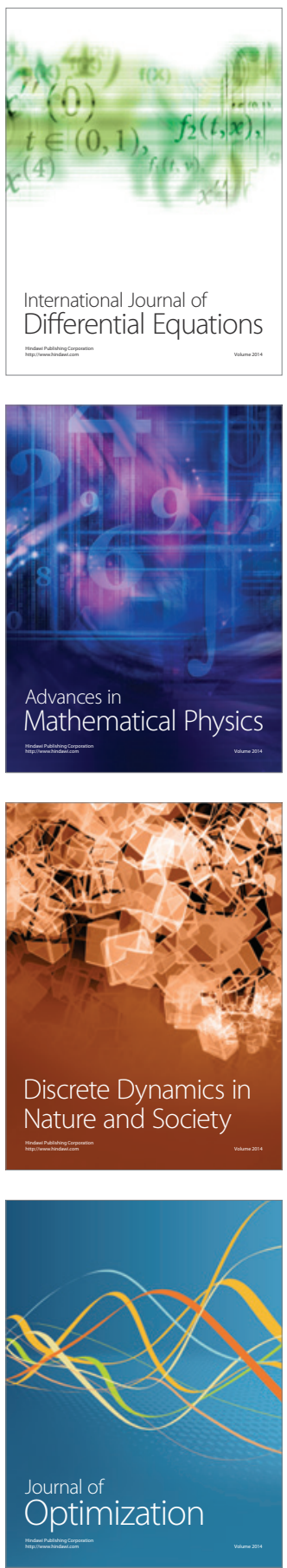